\title{
Audiovisuel d'entreprise en Aquitaine : la mystique et le rationnel
}

Dominique Blin

\section{(2) OpenEdition}

12 Journals

Édition électronique

URL : http://journals.openedition.org/communicationorganisation/1565

DOI : 10.4000/communicationorganisation. 1565

ISSN : $1775-3546$

Éditeur

Presses universitaires de Bordeaux

Édition imprimée

Date de publication : 1 mai 1992

ISSN : 1168-5549

\section{Référence électronique}

Dominique Blin, « Audiovisuel d'entreprise en Aquitaine : la mystique et le rationnel », Communication et organisation [En ligne], 1 | 1992, mis en ligne le 26 mars 2012, consulté le 02 mai 2019. URL : http:// journals.openedition.org/communicationorganisation/1565; DOI : 10.4000/ communicationorganisation. 1565

Ce document a été généré automatiquement le 2 mai 2019.

(c) Presses universitaires de Bordeaux 


\title{
Audiovisuel d'entreprise en Aquitaine: la mystique et le rationnel
}

\author{
Dominique Blin
}

1 Le cinéma industriel n'est pas né avant tous les autres, avec «La sortie des usines Lumière » (1894), comme on le dit souvent. Il n'est pas né en 1910, quand le cinéma se partage entre l'enregistrement strict de la vie, du théatre ou de l'opéra. Ni dans les années 1920-1940, quand le cinéma narratif, muet, puis parlant, se forge un langage propre et se constitue comme art ${ }^{1}$.

2 Il va naître après 1940 avec le «dépassement du taylorisme "², le plan Marshall, des centaines de films de formation ont été alors envoyés en Europe, la diffusion des études d'Elton Mayo et de l'école des Relations Humaines ${ }^{3}$. Longtemps il s'est contenté d'enrichir le domaine du documentaire.

3 Du fait de l'évolution des techniques de prise de vue et des changements intervenus dans le tissu économique on parle maintenant d'audiovisuel d'entreprise. Il s'agit désormais de forger des outils intégrés, des instruments du management et de la gestion.

4 En fait, à l'occasion de la réalisation d'une thèse ${ }^{4}$, nous avons constaté que les produits audiovisuels d'entreprise aquitains s'inscrivaient rarement dans une stratégie générale. Les modes de réalisation et d'exploitation sont fondés sur la mystique de l'image. On s'en remet à elle d'une façon absolue, en la parant de toutes les vertus, comme aux origines du 7 ème art $^{5}$.

5 Ceci dit, la plupart de ces produits ne sont pas construits n'importe comment. Ils peuvent avoir une structure spécifique et constituer un genre : il y a la mystique et le rationnel.

\section{Un discours de mobilisation}

6 Le CREPA (Centre de Relations Publiques Aquitaine) organise depuis 1986 une journée consacrée à l'image et à la communication. L'édition 87 offrait l'avantage de rassembler vingt-sept documents aquitains ${ }^{6}$ représentant des secteurs d'activité très différents. 
7 Nous avons choisi d'analyser dix vidéogrammes d'information promotionnelle commandés par les entreprises suivantes: Aérospatiale, Banque Nationale de Paris, Banque Populaire du Sud-Ouest, Bioluz (laboratoire pharmaceutique); CEA Cesta (centre d'études), Citram (société de transport routier), Lichine (négociant en vin), mairie de Mérignac, établissements Muller (industrie du foie gras) et Sogerma (industrie aéronautique).

Nous avons écarté les simples enregistrements : «Ils ne représentent pas les cas où sont exploitées au maximum les ressources propres au cinéma pour signifier ${ }^{7}$ » et les imitations (vidéogrammes de fiction, vidéogrammes didactiques, etc.) car ils jouent sur des registres non spécifiques.

9 Ces dix vidéogrammes ont, en outre, pour particularité d'avoir été conçus ${ }^{8}$ avec des objectifs et des cibles variés.

\begin{tabular}{|l|l|l|}
\hline La notoriété & Aérospatiale & $\begin{array}{l}\text { BNP } \\
\text { Citram } \\
\text { Mérignac }\end{array}$ \\
\hline L'identité & CEA Cesta & BPSO \\
\hline objectifs & Bioluz & Muller \\
cibles & $\begin{array}{l}\text { Lublichine } \\
\text { restreint }\end{array}$ & $\begin{array}{l}\text { large } \\
\text { public }\end{array}$ \\
\hline
\end{tabular}

10 Tous utilisent deux procédés spécifiques : l'association et la caractérisation.

11 Dans le premier cas, il s'agit de nous présenter alternativement deux éléments de contenu sonore ou visuel (ou deux séries d'éléments), pris dans un rapport de contiguité, afin de composer ou d'agrémenter. Cette opération d'intellection s'inspirera souvent du thème de la détente et de celui du travail.

Dans le deuxième cas, on nous présente successivement un ensemble d'éléments différents : moins pour nous les montrer que pour nous pousser à les considérer comme représentatifs de l'organisation ou de l'entreprise.

13 Au plan syntagmatique (agencement des plans et des séquences) ${ }^{9}$, le premier procédé suscite le syntagme que nous avons appelé « associatif, et un insert (segment autonome, ne comprenant qu'un seul plan, qui a un rôle d'interpolation) équivalent. Dans le vidéogramme de la BNP de nombreux plans : manutentionnaires, ouvriers, techniciens au travail, sont montés en alternance avec des objets appartenant au monde bancaire: 
liasses de billet, tampons, claviers informatiques. Dans le vidéogramme de la BPSO, ce sont des plans présentant des agents actifs de la région : agriculteur, médecin, pilote de ligne, etc. qui alternent avec des plans présentant différents agents de la banque.

Dans le vidéogramme de Bioluz, un plan isolé nous montrant l'océan est suivi par trois autres qui nous montrent la gamme des produits réalisés par le laboratoire. Il s'agit d'un insert associatif, utilisé plusieurs fois, pour introduire une association avec les images du syntagme d'accueil.

Le deuxième procédé suscite le syntagme que nous avons appelé « distinctif » et, lui aussi, un insert équivalent. Dans le document « Portraits et projets » de la mairie de Mérignac on nous propose, successivement, des éléments; moins pour nous les montrer que pour nous pousser à les lier : des entreprises, des lotissements, des équipements culturels et sportifs, un parc de loisirs, des vestiges architecturaux qui sont censés caractériser la commune.

Dans le document Cofinoga et dans celui des établissements Muller un insert présente à plusieurs moments le sigle du commanditaire (insert distinctif).

Les différents plans composant ces deux syntagmes sont généralement liés par utilisation de la coupe franche: liaison directe, ou du fondu enchaîné : court chevauchement d'images donnant à voir en surimpression la fin d'un plan et le début du suivant, le premier disparaissant progressivement au bénéfice du second ${ }^{10}$. La première liaison forge l'accord en accentuant l'impression de réalité, la deuxième confirme cet accord en le rendant effectif, si bien que dans les deux cas c'est l'opération d'association que l'on facilite.

18 Au niveau iconique (articulation des motifs et des éléments signifiants de l'image tels que l'échelle des plans, les mouvements de caméra, l'angle de prise de vue, etc) il y a souvent consolidation des opération d'association et de caractérisation. Le document "L'accord " de la BPSO utilise le passage du flou à la netteté, la plus ou moins grande profondeur de champ pour associer, au sein d'un même plan ou d'un plan à l'autre, ce qui se trouve à l'avant : employé, succursale, et à l'arrière de l'image : client, région. C'est avec le même dessein qu'il emploie le jeu du champ (employé de l'établissement) et du contre-champ (agents représentant les activités de la région) alternativement. Quant aux motifs utilisés pour les opérations de caractérisation, ils sont toujours avantagés par le choix des cadrages et les angles (souvent des contre-plongées) de la prise de vue.

19 Le code de composition sonore (utilisation des bruits, des silences, de la musique et de leurs combinaisons) sert également les deux procédés clefs. La bande-son du vidéogramme de la BNP est émaillée de bruits infinitésimaux, caractéristiques du monde bancaire: coups de tampon, crissements de plumes, froissements de billets, etc. La musique expressive est souvent préférée à la musique d'accompagnement car elle permet l'association d'idée : guitare hawaïenne pour lier les produits Bioluz et la mer; solo de violoncelle pour conférer mystère et tradition aux vins de chez Lichine.

20 Une association particulièrement servie par les bruits « off » « qui émanent d'une source invisible située dans un autre temps et/ou un autre lieu que l'action montrée dans l'image $»^{11}$ : mouettes et cornes de navire chez Citram, chants d'oiseaux à la Sogerma.

21 Enfin, il y a confirmation des opérations d'association et de caractérisation au plan de l'articulation entre images et paroles (aspects du code de composition audiovisuelle) du fait de la très nette domination des cas de simultanéité entre les deux. Le commentaire off 
simultané et très abondant sert souvent à préciser le sens des images (fonction d'ancrage) quand elles opèrent pour associer et pour caractériser.

Ces deux opérations, si souvent provoquées, sont caractéristiques du vidéogramme d'information institutionnelle. Elles sont conjuguées avec d'autres, moins spécifiques : on montre aussi, on suggère, on fait allusion, etc. de façon à susciter chez le destinataire différents types de comportements qui tous concourent au même but : mobiliser.

Chaque catégorie, chaque genre audiovisuel se distingue par une inclination particulière. Ainsi, par exemple, le document didactique privilégie l'implication ${ }^{12}$. Ce qui spécifie le plus l'intention promotionnelle c'est la mobilisation. Les dix produits analysés permettent de dégager un véritable code de la mobilisation qui agit aux différents niveaux de la structure filmique.

\begin{tabular}{|c|c|c|c|c|}
\hline \multirow{2}{*}{$\begin{array}{l}\text { Modalités } \\
\text { (comportements } \\
\text { du destinataire } \\
\text { visés) }\end{array}$} & \multicolumn{4}{|c|}{ Intervenant au plan } \\
\hline & $\begin{array}{l}\text { du message } \\
\text { global }\end{array}$ & du son & de lïmage & $\begin{array}{l}\text { des rapports } \\
\text { image/son }\end{array}$ \\
\hline I Attirer & $\begin{array}{l}\text { Rôle de } \\
\text { l'interprètre et } \\
\text { de linterviewé } \\
\text { Compositoin } \\
\text { Rôle de } \\
\text { l'interviewé }\end{array}$ & $\begin{array}{l}\text { Changements } \\
\text { musicaux } \\
\text { Changements } \\
\text { de rythme } \\
\text { et/ou } \\
\text { dintensite } \\
\text { musicale }\end{array}$ & $\begin{array}{l}\text { Code du regard et de } \\
\text { l'exclusivité } \\
\text { Image en médaillon, } \\
\text { image réduite } \\
\text { Zoom et gros plans } \\
\text { Montage rapide }\end{array}$ & $\begin{array}{c}\text { Variation dans les } \\
\text { rapports }\end{array}$ \\
\hline $\begin{array}{l}\text { II Aiguiser la } \\
\text { curiosité }\end{array}$ & Composition & $\begin{array}{c}\text { Bruits } \\
\text { d'ambiance }\end{array}$ & 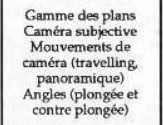 & $\begin{array}{l}\text { Musique "on" } \\
\text { Bruitage en } \\
\text { avance }\end{array}$ \\
\hline $\begin{array}{l}\text { III Faciliter la } \\
\text { compréhension }\end{array}$ & $\begin{array}{l}\text { Rôle de } \\
\text { linterprête }\end{array}$ & $\begin{array}{c}\text { Rôle du } \\
\text { commentaire } \\
\text { Bruitages } \\
\text { sélectionnés }\end{array}$ & $\begin{array}{l}\text { Images de synthese } \\
\text { Gros plans } \\
\text { Surimpressions } \\
\text { image treter } \\
\text { Graphique } \\
\text { Nettete et flou }\end{array}$ & $\begin{array}{l}\text { Simultaneitect ou } \\
\text { avance du } \\
\text { commentaire }\end{array}$ \\
\hline $\begin{array}{l}\text { IV Fournir des } \\
\text { ééments de } \\
\text { détente et de } \\
\text { confort }\end{array}$ & Composition & 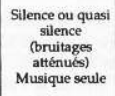 & $\begin{array}{l}\text { Plan fixe long } \\
\text { Longueur des } \\
\text { panoramiques ou } \\
\text { travellings ou }\end{array}$ & $\begin{array}{l}\text { Image avec son } \\
\text { feutré }\end{array}$ \\
\hline $\begin{array}{l}\text { V S Susciter } \\
\text { lenchantement } \\
\text { et l'harmonie }\end{array}$ & Composition & $\begin{array}{l}\text { Bruitages } \\
\text { atténués ou } \\
\text { sélectionnés } \\
\text { Rôle de la } \\
\text { musique }\end{array}$ & $\begin{array}{l}\text { Mouvements relayés } \\
\text { Image et images dans } \\
\text { limage } \\
\text { Vue aérienne, rotation } \\
\text { Surimpression } \\
\text { Image encadrée } \\
\text { Mouvements } \\
\text { d'accompagnement }\end{array}$ & $\begin{array}{l}\text { Synchronismes: } \\
\text { mouvements de } \\
\text { limage par rap- } \\
\text { port a la musique } \\
\text { dhangements } \\
\text { d'image par } \\
\text { rapport al ia } \\
\text { musique }\end{array}$ \\
\hline $\begin{array}{l}\text { VI Développer } \\
\text { la confiance }\end{array}$ & $\begin{array}{c}\text { Rôle de } \\
\text { linterprète }\end{array}$ & $\begin{array}{l}\text { Changements } \\
\text { de voix- Ton } \\
\text { du commentaire } \\
\text { Force de la } \\
\text { musique }\end{array}$ & $\begin{array}{l}\text { Mouvements } \\
\text { d'accompagnement } \\
\text { Plans rapporchés et } \\
\text { gros plans } \\
\text { Caméra subjective } \\
\text { Personnage seul }\end{array}$ & Simultanéité \\
\hline $\begin{array}{l}\text { VII Favoriser } \\
\text { l'adhésion }\end{array}$ & $\begin{array}{c}\text { Rôle de } \\
\text { l'interprète }\end{array}$ & $\begin{array}{c}\text { Leiturotiv } \\
\text { musical } \\
\text { Repetition des } \\
\text { bruitages }\end{array}$ & 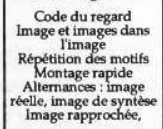 & $\begin{array}{l}\text { Bruitages "on" } \\
\text { Musique en } \\
\text { avanoe }\end{array}$ \\
\hline
\end{tabular}

\section{Des pratiques intuitives}

Nous avons rencontré les dix commanditaires des produits sélectionnés et dépouillé les questionnaires remplis par seize prestataires aquitains ${ }^{13}$ pour connaître les modes de réalisation et d'exploitation des produits audiovisuels d'entreprise.

La prise de décision concernant la commande est le plus souvent motivée par la proximité d'une manifestation : foire, salon, congrès, convention, etc. Elle est justifiée en deuxième lieu, par la volonté de présenter une nouveauté technique ou économique. Dans les deux cas, il s'agit plutôt d'une attitude occasionnelle et non pas d'une action liée à telle ou telle phase d'un plan de communication. Plusieurs commanditaires nous ont dit avoir opéré dans l'urgence.

26 Les objectifs ont été généralement clairement définis au départ mais ils ne sont pas toujours atteints. Soit que le document ait été façonné au détriment de l'un d'eux: la sensibilisation voulue à l'aspect « grand tourisme » de l'entreprise Citram est loin d'être 
privilégiée par le vidéogramme; soit que la facture générale ait finalement contrariée l'essentiel : l'image de prestige et de qualité propre au vin n'est pas servie par le produit Lichine qui passe d'un cliché à l'autre.

27 Ceci dit, ils sont souvent nombreux. Atteindre trois objectifs constitue (ex. Bioluz) un maximum. Au-delà, le résultat ne peut pas être convaincant. C'est le cas du produit de la BPSO auquel le bulletin d'information des sociétaires ${ }^{14}$ attribue officiellement les intentions suivantes : créateurs de son projet à un professionnel de la production dont le métier est de connaitre et de diriger des auteurs. Cependant, de nombreux commanditaires choisissent de lancer des appels d'offres $»^{15}$. L'Aquitaine compte peu de producteurs stricto-sensu ; c'est-à-dire de maîtres d'œuvre capables de représenter le commanditaire, de choisir les collaborateurs, de prendre la responsabilité de la réalisation et de l'exploitation du produit. En conséquence on procède directement au choix d'un réalisateur. Il est souvent sélectionné à partir d'un échantillon réduit (une seule réponse à l'appel d'offres des établissements Muller), ou sur recommandation de services non spécialisés : agence de publicité, service commercial. Quand le choix est plus large, le coût proposé pour l'opération constitue, de loin, le premier critère de sélection. Le commanditaire est sensible aussi à la qualité de la relation et à la rapidité d'exécution. Le degré de créativité n'est considéré qu'ensuite. plus attentif, il tient à superviser les documents écrits : trames ou scénarii. Il composera éventuellement les commentaires mais il s'en remet complètement au réalisateur pour l'image. surtout utilisés dans l'enceinte des établissements, lors des déplacements auprès des clients et des fournisseurs, à l'occasion des manifestations pour lesquelles, souvent, ils ont été conçus.

À quelques détails près, la satisfaction des commanditaires consultés est totale. Ces produits, c'est sûr, ont eu un effet d'entraînement constaté généralement du fait de l'augmentation des ventes, du nombre de contrats ou de la hausse du chiffre d'affaires. Mais les opérateurs (prestataires et commanditaires) considèrent aussi qu'ils ont été bien 
reçus. Le public aurait été conquis même si on regrette une séquence, un élément de la composition (BPSO, Bioluz, Mérignac) ou si on constate des absences (Lichine).

Quant à savoir comment ils ont été lus et si les éléments d'information prévus au départ ont été transmis ? ... Ces documents existent, produisent un certain effet; ils sont donc satisfaisants.

La technique vidéo est partout saluée. On la considère pour sa commodité (tournage, utilisation, duplication), pour l'illusion qu'elle entretient (parenté avec le film), pour les tours qu'elle recèle (la magie de l'image de synthèse). Rapide, souple, dynamique, on s'y fie ; elle est taillée pour informer, il n'est pas question d'en douter. Nous sommes bien en pleine mystique de l'image.

D'un point de vue structurel, les vidéogrammes aquitains d'information promotionnelle, sont sur la voie de la maturité. Il reste, pour les démarches, à quitter l'ère du merveilleux.

\section{NOTES}

1. André BAZIN, Qu'est ce que le cinéma? Chapitre VII: «l'évolution du Langage Cinématographique ", Paris, Ed. du Cerf, 1985 (Coll, 7ème Art).

2. Gérard LEBLANC, Quand l'entreprise fait son cinéma: "Les commencements du cinéma industriel », Paris, Cinéthique/ Presses Universitaires de Vincennes, 1983. p. 21-28.

3. Jacques LEGRES et Daniel PEMATIN, Pratique des Relations Humaines dans l'entreprise : «L'évolution de conditions de travail », Paris, Ed. d'organisation, 1981, p. 59-91.

4. Dominique BLIN, Rencontre d'un discourt et d'un mode d'expression: information institutionnelle et vidéo grammes dans le contexte aquitain. Thèse de doctorat de l'Université de Bordeaux 3, novembre 1989.

5. Elie FAURE, Fonction du cinéma: «Introduction à la mystique du Cinéma ", Paris, Ed. Gonthier, 1964 (Coll. Médiation), p. 48-68.

6. C'est à dire vingt sept documents commandes par des entreprises ou établissements installés en région Aquitaine mais réalisés par des prestataires de la France entière.

7. Geneviève JAC-QUINOT, Image et pédagogie, Paris, P.UF 1977, p. 42.

8. Renseignements fournis par les commanditaires.

9. Christian Metz, Essai sur la signification au cinéma. Paria Klincksieck, t.1 et t. 2, 1971 et 1973.

10. Daniel Percheron lectures du film: "Ponctuation », Paris, Ed. Albatros, 1976, (Coll, Ça/ Cinéma),

11. Michel CHION,

Le son au cinéma,

Parte, Cahier» du cinéma/Ed. de l'Etoile, 1985, p. 32.

12. Geneviève JACQUINOT, op. cit., p. 69.

13. Onze en Gironde, trois dans les Pyrénées Atlantiques, deux en Lot et Garon ne sur trente : dix neuf en Gironde, neuf dans les Pyrénées-Atlantiques et deux en Lot et Garonne recenses en Aquitaine par le «Presta'Com », annuaire des prestataires et des commanditaires du Grand Sud, Toulouse, 1989.

14. Le Sociétaire, $n^{\circ} 30$, avril 1988, P.8. 
15. François COHEN SEAT, Anne-Marie FALCONNET, Le guide du producteur, Paris, Ed. Dixit, 1987, p. 21.

\section{RÉSUMÉS}

Les audiovisuels d'entreprise aquitains utilisent deux procédés spécifiques : l'association et la caractérisation. En agissant aux différents niveaux de la structure filmique ces deux procédés concourent à forger un discours de mobilisation propre à l'intention promotionnelle. La forme et la matière de ces produits, construits de façon rationnelle, permettent de parler de l'émergence d'un genre.

Les modes de réalisation et d'exploitation relèvent par contre des pratiques intuitives. La commande est souvent passée à l'occasion, les cibles ne sont pas toujours bien connues. Ces documents existent ; ils sont donc satisfaisants. C'est la mystique de l'image.

\section{AUTEUR}

\section{DOMINIQUE BLIN}

Dominique Blin, Maître de conférences à l'IUT Michel de Montaigne de Bordeaux 3, est responsable de la filière communication d'entreprise. Il enseigne les méthodes de la communication, analyse et conçoit des opérations à caractère professionnel. Il a publié plusieurs articles en collaboration avec l'Université de Lille 3. Il participe aux activités du GREC, devenu GRECO, depuis sa création. 